\title{
LIPOSOMES EMBEDDED WITH DIFFERENTIATING FACTORS AS A NEW STRATEGY FOR ENHANCING DPSC OSTEOGENIC COMMITMENT
}

\author{
M. Gallorini ${ }^{1,3}$, R. Di Carlo ${ }^{2}$, S. Pilato ${ }^{1}$, A. Ricci ${ }^{1}$, H. Schweikl ${ }^{3}$, A. Cataldi ${ }^{1}$, A. Fontana ${ }^{1}$ and S. Zara ${ }^{1, *}$ \\ ${ }^{1}$ Department of Pharmacy, University “G. d'Annunzio" Chieti-Pescara, Chieti, Italy \\ ${ }^{2}$ Department of Medical, Oral and Biotechnological Sciences, University "G. d'Annunzio" Chieti-Pescara, \\ Chieti, Italy \\ ${ }^{3}$ Department of Conservative Dentistry and Periodontology, University Hospital Regensburg, \\ University of Regensburg, Regensburg, Germany
}

\begin{abstract}
Human dental pulp stem cell (DPSC) differentiation toward the osteoblastic phenotype is enhanced when culture media are supplemented with differentiating factors, i.e. ascorbic acid, $\beta$-glycerophosphate and dexamethasone. Liposomes, spherical vesicles formed by a phospholipid bilayer, are frequently used as carriers for drugs, growth factors and hydrophobic molecules. The aim of this work was to speed up DPSC commitment to the osteogenic lineage by embedding differentiating factors within liposomes. Firstly, liposomes were prepared by rehydrating a phospholipidic thin film and characterised in terms of dimensions. Secondly, liposome-exposed DPSCs were characterised by their immunophenotypic profile. Levels of CD90 were significantly decreased in the presence of liposomes filled with ascorbic acid, $\beta$-glycerophosphate and dexamethasone (Lipo-Mix) with respect to normal differentiation medium (DM), while CD73 and CD29 expression were enhanced, suggesting osteogenic commitment. Additionally, an appreciable extracellular matrix deposition is detected. Thirdly, the Lipo-Mix formulation better increases alkaline phosphatase activity and levels of Collagen I secretion with respect to DM. In parallel, the new liposome formulation is capable of decreasing the release of $\mathrm{H}_{2} \mathrm{O}_{2}$ and of triggering a precocious antioxidant cell response, redressing the redox balance required upon mesenchymal stem cell commitment to osteogenesis. It can be therefore hypothesised that Lipo-Mix could represent a suitable tool for clinical regenerative purposes in the field of tissue engineering by speeding up DPSC osteogenic commitment, mineralised matrix deposition and remodelling.
\end{abstract}

Keywords: Liposomes, DPSC differentiation, ascorbic acid, dexamethasone, $\beta$-glycerophosphate, oxidative stress.

*Address for correspondence: Susi Zara, via dei Vestini 31, University "G. d'Annunzio" Chieti-Pescara, 66100, Chieti, Italy.

Telephone number: +390871 3554507 Email: susi.zara@unich.it

Copyright policy: This article is distributed in accordance with Creative Commons Attribution Licence (http://creativecommons.org/licenses/by-sa/4.0/).

\section{List of Abbreviations}

ALP

ANOVA

ARS

Asc

ATP

BSA

BSPII

CD

CD105

CD29

CD45

CD73
CD90
Col1
Dex
DLS
DM
DMSO
DPSCs
ECL
ECM
EPC
Erk1/2
FACS
FBS

purine 5-prime-nucleotidase

Thy-1 membrane glycoprotein

collagen type I

dexamethasone

dynamic laser light scattering

differentiation medium

dimethyl sulphoxide

dental pulp stem cells

enhanced chemiluminescent

extracellular matrix

egg yolk phosphatidylcholine

extracellular signal-regulated kinase $1 / 2$

fluorescence-activated cell sorting

foetal bovine serum 


$\begin{array}{ll}\text { FITC } & \text { fluorescein isothiocyanate } \\ \text { FSC } & \text { forward scatter } \\ \text { GPI } & \text { glycophosphatidylinositol } \\ \text { HA } & \text { horseradish peroxidase } \\ \text { HRP } & \text { integrated optical intensity } \\ \text { IOI } & \text { mitogen-activated protein kinase } \\ \text { MAPK } & \text { median fluorescence intensities } \\ \text { MFI } & \text { mesenchymal stem cells } \\ \text { MSC } & \text { optical density } \\ \text { OD } & \text { phosphate buffered saline } \\ \text { PBS } & \text { polydispersity index } \\ \text { PDI } & \text { phycoerythrin } \\ \text { PE } & \text { active Erk1/2 } \\ \text { pErk1/2 } & \text { phenylmethylsulphonyl fluoride } \\ \text { PMSF } & \text { p-nitrophenyl phosphate } \\ \text { pNPP } & \text { 1-palmitoyl-2-oleyl-sn-glycero-3- } \\ \text { POPC } & \text { phosphocoline } \\ & \text { reactive oxygen species } \\ \text { ROS } & \text { runt-related transcription factor 2 } \\ \text { Runx2 } & \text { standard deviation } \\ \text { SD } & \text { sodium dodecyl sulphate } \\ \text { SDS } & \text { side scatter } \\ \text { SSC } & \text { time of cell detachment } \\ \text { T0 } & \text { transforming growth factor- } \beta \\ \text { TGF- } \beta & \text { wingless and int-1 } \\ \text { Wnt } & \alpha \text {-modified minimum essential } \\ \alpha-M E M & \text { medium } \\ \beta \text {-Gly } & \text { superficial charge } \\ \zeta \text {-potential } & \\ & \end{array}$

\section{Introduction}

Phospholipids are molecules with amphiphilic properties because they include in their structure both hydrophobic and hydrophilic chemical groups. As a result of their amphiphilicity, when immersed in an aqueous medium, phospholipids are able to selfassemble into micelles, lipid bilayers and liposomes (Fahy et al., 2008).

Liposomes, initially discovered by Bangham in the 60s (Bangham et al., 1965), are spherical vesicles obtained due to the tendency of the hydrophilic polar heads of phospholipids to expose towards water and the hydrophobic phospholipidic tails to cluster together - forming a bilayer that encloses an aqueous core. Considering their several advantageous properties, numerous studies have examined liposomes as possible nano-carriers. Liposomes possess a versatile structure allowing loading of a huge range of bioactive compounds both in their inner compartment and within the lipidic bilayer (Almeida and Souto, 2007; Gomes-da-Silva et al., 2012; Moura et al., 2012). Their properties include very-low cytotoxicity and full biodegradability (Moreira et al., 2002), as well as an appreciable capability to augment the efficacy of bioactive molecules (Akbarzadeh et al., 2013). Liposomes have been widely used as drug-release systems thanks to both their carrying capability and ability to simultaneously protect bioactive agents such as fragmented or whole antibodies (Cortese et al., 2020; Moura et al., 2012), fluorophores (Singh et al., 2001; Zappacosta et al., 2014), growth factors or other molecules (Monteiro et al., 2015; Sinjari et al., 2019; Viale et al., 2016; Zappacosta et al., 2019a; Zappacosta et al., 2019b).

Tissue regeneration is based on stem-cell differentiation towards specific lineages. It generally occurs if an adequate delivery of growth/ differentiation factors is guaranteed to the target cells. In this context, biocompatible nano-carriers - such as liposomal formulations - are ideal candidates for directly delivering signalling molecules and/or growth/differentiation factors, as has already been demonstrated (Lee et al., 2011; Santo et al., 2013a; Santo et al., 2013b).

MSCs are adult stem cells identified by: their adherence to plastic; the expression of cell surface markers, including CD90, CD73, CD105 and CD29; lack of the expression of hematopoietic markers; no immunogenic effect; and their ability to replace damaged tissues (Maleki et al., 2014). Due to their behaviour in terms of proliferation, differentiation, and adherence to plastic, they have been widely used in experimental models of regenerative studies. DPSCs are identified as MSCs. Human DPSCs, isolated from dental pulp, represent an appreciable source of undifferentiated stem cells with a higher clonogenic capability compared to bone marrow stem cells (Brożek et al., 2017). Moreover, dental pulp is an accessible surgical site facilitating easy sample harvesting and extraction of cells. DPSCs can easily progress from undifferentiated cells to secretory odontoblasts/osteoblasts, thus allowing the deposition of a mineralised extracellular matrix (Goto et al., 2016; Graziano et al., 2008).

The standard protocol for in vitro stem-cell differentiation towards the osteoblastic lineage requires continuous exposure to a cocktail of specific differentiation factors, added to the standard medium for at least $21 \mathrm{~d}$; usually Asc, Dex and $\beta$-Gly are included (Song et al., 2009). Dex induces Runx2 expression, the Asc leads to the increased secretion of Col1, which in turn leads to increased Col1/ $\alpha 2 \beta 1$ integrin-mediated intracellular signalling, and $\beta$-Gly serves as a source of phosphate in HA, simultaneously influencing intracellular signalling molecules (Langenbach and Handschel, 2013). Moreover, it has been reported that the molecular pathways underlying the MSC osteoblastic differentiation are tightly regulated by ROS. In parallel, high levels of ROS lead to oxidative stress-related apoptosis and cell cycle arrest in MSCs (Atashi et al., 2015).

Considering that a challenging target of regenerative medicine is to efficiently promote the osteoblastic differentiation of stem cells, this study aimed at using liposomes as nano-carriers systems to deliver all the 3 differentiating factors (Asc, Dex and $\beta$-Gly) to DPSCs. The efficiency of this LipoMix formulation in triggering DPSCs commitment to the osteogenic lineage was evaluated in terms of 
osteogenesis speed, expression of specific markers and deposition of mineralised matrix when compared with differentiating factors commonly dissolved and diluted within the culture medium. The relationship between DPSC differentiation capacity and ROS generation was also investigated.

\section{Materials and Methods}

\section{Preparation of liposomes}

In order to form a thin lipid film on the inside wall of a flask, appropriate aliquots of POPC (Avanti Polar Lipids, Inc, Alabaster, AL, USA) and cholesterol (Sigma-Aldrich, St. Louis, MO, USA) dissolved in chloroform to obtain a final concentration of $10 \mathrm{mmol} / \mathrm{L}$ (with ratio of $90: 10$ ), was put in a roundbottomed flask and dried using a rotary evaporator under reduced pressure at $40{ }^{\circ} \mathrm{C}$. The phospholipid film was kept at $4{ }^{\circ} \mathrm{C}$ overnight, before rehydration with PBS (pH 7.4). Empty liposomes, without differentiating factors, were obtained by rehydration of the film in $2.5 \mathrm{~mL}$ of PBS ( $\mathrm{pH} 7.4$ ). Liposomes embedding hydrophilic differentiating factors, i.e. Asc and $\beta$-Gly, were obtained by rehydrating the phospholipid film with a solution containing $2 \mathrm{~mL}$ of PBS, $250 \mu \mathrm{L}$ of Asc and $250 \mu \mathrm{L}$ of $\beta$-Gly. Liposomes enriched with Dex were obtained by rehydrating the film with a solution containing $2.25 \mathrm{~mL}$ of PBS added with $250 \mu \mathrm{L}$ of Dex in DMSO (Sigma-Aldrich). After $30 \mathrm{~min}$ of rehydration, the liposomes were sonicated at $37 \mathrm{kHz}$ (Elmasonic $\mathrm{P} 60 \mathrm{H}, 180 \mathrm{~W})$ for $30 \mathrm{~min}$. For the in vitro test, liposomes were sterilised under an UV lamp for $2 \mathrm{~h}$ (Hera Safe, distance 20cm, $254 \mathrm{~nm}$ wavelenght, 15W power). In order to obtain a liposomal suspension with the 3 differentiating factors, the same amount of the 2 latter liposomal suspensions, containing the hydrophilic and hydrophobic differentiating factors, respectively, were mixed. $\beta$-Gly, Asc and Dex, (50 mmol/L, $1 \mathrm{mmol} / \mathrm{L}$ and $100 \mathrm{nmol} / \mathrm{L}$, respectively) were included in the liposomal suspension.

Liposomes were characterized in terms of size and $\zeta$-potential by using a dynamic laser light scattering apparatus (Brookhaven Instrument Corp., Holtsville, NY, USA).

\section{Preparation of medium}

Three different culture conditions were investigated: DM, Lipo-Empty and Lipo-Mix. DM was obtained by supplementing complete $\alpha$-MEM with osteogenic differentiating factors (Dex: $10 \mathrm{nmol} / \mathrm{L}, \beta-\mathrm{Gly}$ : $5 \mathrm{mmol} / \mathrm{L}$, Asc: $0.1 \mathrm{mmol} / \mathrm{L}$ (Sigma-Aldrich). LipoEmpty was made up of empty liposomes diluted $1: 10 \mathrm{v} / \mathrm{v}$ with DM. Lipo-Mix consisted of liposomes embedded with the 3 osteogenic differentiating factors (Dex, $\beta$-Gly and Asc), dispersed $1: 10 \mathrm{v} / \mathrm{v}$ in $\alpha$-MEM. The final concentrations of the differentiating factors in Lipo-Mix were $10 \mathrm{nmol} / \mathrm{L}, 5 \mathrm{mmol} / \mathrm{L}$ and $0.1 \mathrm{mmol} / \mathrm{L}$, respectively.

\section{Cell culture}

DPSCs (Lonza Group Ltd., Basel, Switzerland) were cultured, at $37^{\circ} \mathrm{C}$ with $5 \% \mathrm{CO}_{2}-$ in $\alpha$-MEM (SigmaAldrich), supplemented with $10 \%$ FBS, 1\% penicillin/ streptomycin (Euroclone S.p.A., Milan, Italy) - under DM, Lipo-Empty or Lipo-Mix conditions for up to $21 \mathrm{~d}$. At each experimental time $(1,3,7,14$ and $21 \mathrm{~d})$ samples were collected and processed for experimental tests.

\section{Immunophenotyping by flow cytometry}

DPSCs were seeded at passage $5\left(0.5 \times 10^{5}\right.$ cells/well $)$ in 6-well plates and allowed to adhere for $24 \mathrm{~h}$. After having detached the cells at T0 and exposed cultures to DM, Lipo-Empty and Lipo-Mix conditions for 3, 5 and $7 d$, respectively, DPSCs were harvested with Accutase ${ }^{\circledR}$ solution (Sigma Aldrich) and collected by centrifugation $(450 \times g)$ in the cold. The cell pellets were washed once with FACS binding buffer $(5 \mathrm{mmol} / \mathrm{L}$ Tris- $\mathrm{HCl}, 0.5 \mathrm{mmol} / \mathrm{L}$ ethylenediaminetetraacetic acid, $1 \mathrm{mmol} / \mathrm{L} \mathrm{NaCl}$ in deionised water, $\mathrm{pH}$ 7.4). Cells were then incubated with fluorochromeconjugated antibodies (1: 50 dilutions) in $50 \mu \mathrm{L}$ of FACS buffer for 15 min in the dark at room temperature. Samples were stained separately in each single screening tube with anti-CD45-FITC (eBioscience, Waltham, MA, USA), anti-CD90-FITC (BD Pharmigen, San Jose, CA, USA), anti-CD73-PE (R\&D System, Minneapolis, MN, USA), anti-CD105PE and anti-CD29-PE (both from Ancell Corporation, Stillwater, MN, USA). After that, volumes were doubled by adding fresh FACS-binding buffer and samples were centrifuged at $450 \times g$ for $5 \mathrm{~min}$ in the cold, to remove excess antibody. Finally, 20,000 events were run in a CytoFlex cytometer (Beckman Coulter, Brea, CA, USA) equipped with a $488 \mathrm{~nm}$ laser and FL-1 and FL-2 in a linear mode. Relative fluorescence emissions of cells, gated by means of their FSC and SSC, were analysed with the CytExpert Software (Beckman Coulter). MFI ratios, for the FITC and the PE fluorescence, were also determined for the mouse isotype controls. Individual values obtained from three independent experiments $(n=3)$ were summarised as means and SD.

\section{ARS staining}

DPSCs were seeded as described above for the immunophenotyping. After 1, 3, 7, 14 and $21 \mathrm{~d}$ of culture, medium was removed and the cell monolayer washed in PBS with $\mathrm{Ca}$ and $\mathrm{Mg}$. Cells were fixed for 15 min with $4 \%$ paraformaldehyde at room temperature and then rinsed $2 \times$ with deionised water. ARS solution (Sigma-Aldrich), at a final concentration of $40 \mathrm{mmol} / \mathrm{L}$, was added in each well and incubated for $20 \mathrm{~min}$ at room temperature, followed by several washes with deionised water to remove excess dye. Calcium nodules, highlighted by ARS, which appeared as bright orange-red stained areas, were acquired using a Leica DMIL inverted light microscope at $20 \times$ magnification. 


\section{ALP activity}

At the established exposure times, cell supernatants were collected from 6-well plates (cell seeding $0.5 \times 10^{5} \mathrm{DPSC} /$ well $)$ and ALP activity was determined using an ALP assay kit (Colorimetric) (Abcam, Cambridge, UK). The kit uses pNPP as a phosphatase substrate that turns yellow $(\lambda \max =405 \mathrm{~nm})$ when dephosphorylated by ALP. $80 \mu \mathrm{L}$ of each supernatant were collected and loaded in duplicate in a 96-well clear flat bottom treated-culture microplate (Falcon, Corning, NY, USA) and $50 \mu \mathrm{L}$ of $5 \mathrm{mmol} / \mathrm{L} \mathrm{pNPP/}$ well were added afterwards. After $1 \mathrm{~h}$ of incubation at room temperature in the dark, $20 \mu \mathrm{L}$ of the provided stop solution were added in each well. The absorbance was measured at $405 \mathrm{~nm}$ by means of a microplate reader (Multiskan GO, Thermo Scientific, Inc., Waltham, MA, USA). The ALP activity (mU/mL/ $\mathrm{min}$ ) was calculated by following the manufacturer's specifications and values and expressed as the fold increase on the DM sample at day 1.

\section{Cell lysis and protein extraction}

After 1, 3 and $7 \mathrm{~d}$ of treatment, as previously described, cells were detached from the 6-well plates by trypsinisation, collected in cold PBS by centrifugation at $450 \times g$ and pellets were then washed $2 \times$ with cold PBS. Lysis buffer $(70 \mu \mathrm{L})$ containing a cocktail of protein inhibitors [PBS, containing: $1 \%$ IGEPAL ${ }^{\oplus}$ CA-630, $0.5 \%$ sodium deoxycholate, $0.1 \%$ SDS, $10 \mathrm{mg} / \mathrm{mL}$ PMSF, $1 \mathrm{mg} / \mathrm{mL}$ aprotinin, $10 \mathrm{mM}$ sodium orthovanadate and $50 \mu \mathrm{g} / \mathrm{mL}$ leupeptin (Sigma-Aldrich)] was added to the pellets and samples were kept on ice for $30 \mathrm{~min}$. After that, samples were resuspended and set on ice for an additional $30 \mathrm{~min}$. Cell lysates were centrifuged for $15 \mathrm{~min}$ at 20,000 $\times g$ and supernatants containing proteins as the whole cell fraction were collected. Protein concentration was determined through a bicinchoninic acid assay (QuantiPro ${ }^{\mathrm{TM}}$ BCA Assay kit for $0.5-30 \mu \mathrm{g} / \mathrm{mL}$ protein, Sigma-Aldrich, Milan, Italy) following the manufacturer's instructions. The amount of protein in each sample was calculated from a standard curve, established with the BSA provided with the kit, by means of the Prism 5.0 software (GraphPad, San Diego, CA, USA).

\section{Immunoblotting}

DPSCs cell lysates $(20 \mu \mathrm{g} / \mathrm{sample})$ wereelectrophoresed on a 4-20\% SDS-PAGE gel (ExpressPlus ${ }^{\mathrm{TM}} 10 \times 8$, GenScript Biotech Corporation, Nanjing, China) and transferred to nitrocellulose membranes. The latter were blocked in $5 \%$ of non-fat milk or $5 \%$ BSA, $10 \mathrm{mmol} / \mathrm{L}$ Tris pH 7.5, $100 \mathrm{mmol} / \mathrm{L} \mathrm{NaCl}$, $0.1 \%$ Tween 20 and probed overnight at $4{ }^{\circ} \mathrm{C}$ under gentle shaking with mouse monoclonal anti- $\beta$ actin (Sigma-Aldrich) (primary antibody dilution $1: 10,000)$, mouse monoclonal anti-pro-collagen 1A1 (primary antibody dilution $1: 200$ ), mouse monoclonal anti-BSPII (primary antibody dilution $1:$ 200) antibodies (Santa Cruz Biotechnology, Santa
Cruz, CA, USA), rabbit anti-p44/42 MAPK and anti-phosho-p44/42 MAPK (Erk 1/2 and p-Erk 1/2) monoclonal antibodies (primary antibodies dilution $1: 1,000$ ) (Cell Signaling Technology, Danvers, MA, USA). Subsequently, the membranes were incubated in the presence of specific HRP-conjugated IgG secondary antibodies. Immunoreactive bands were revealed using the ECL detection system (LiteAblot Extend Chemiluminescent Substrate, EuroClone S.p.a., Milan, Italy) and underwent densitometry. Densitometric values, expressed as IOI, were estimated using a ChemiDoc ${ }^{\mathrm{TM}}$ XRS system and the QuantiOne 1-D analysis software (BIORAD, Richmond, CA, USA). Values obtained were normalised based on densitometric values of internal $\beta$-actin. Results are expressed as mean values \pm SD.

\section{Hydrogen peroxide release}

At the suitable exposure times, cell supernatants were collected as previously described for the analysis of ALP activity. The quantitative determination of $\mathrm{H}_{2} \mathrm{O}_{2}$ released in supernatants was performed by means of a $\mathrm{H}_{2} \mathrm{O}_{2}$ colorimetric detection kit (cat. no. ADI-907015, Enzo Life Sciences, Inc., Farmingdale, NY, USA). For each sample, $50 \mu \mathrm{L}$ of supernatant/well were loaded, in duplicate, in a half-area microtiter plate, and $100 \mu \mathrm{L}$ of colour reagent were added in each well. The contents of the wells were mixed by shaking the plate for $10 \mathrm{~s}$ and then incubating it for $30 \mathrm{~min}$ at room temperature. The OD was measured at $550 \mathrm{~nm}$ by means of a spectrophotometer (Multiskan GO). The results were calculated by subtracting the average blank OD from the average OD, for each sample, and then interpolating these values with a standard curve - following the manufacturer's instructions.

\section{ELISA analysis of Col1 secretion}

Absolute amounts of Col1 secreted in supernatants harvested from 6-well plates from 1 to $21 \mathrm{~d}$ of culture were detected using a human Col1 ELISA kit (cat. no. ACE-EC1-E105-EX, Cosmo Bio Co., Ltd., Tokyo, Japan). Samples were pipetted into suitably coated wells, then the blue conjugate and the yellow antibody were added. After $2 \mathrm{~h}$ of incubation at room temperature on a plate shaker, wells were washed and then incubated with the pNPP substrate for $45 \mathrm{~min}$. Finally, a stop solution was added, and absorbance was measured at $450 \mathrm{~nm}$ using a microplate reader (Multiskan GO). The concentration of Col1 $(\mu \mathrm{g} / \mathrm{mL})$ was calculated using a standard curve generated with specific standards provided by the manufacturers by means of the Prism 5.0 software (GraphPad).

\section{Statistical analysis}

Statistical analysis was established by one-way ANOVA, followed by Tukey's post-hoc test using the Prism 5.0 software (GraphPad). Results were expressed as means $\pm \mathrm{SD}$. Values of $p<0.05$ were considered statistically significant. 


\section{Results}

\section{Characterisation of liposomes}

The obtained liposomes were characterised in terms of dimensions by DLS. The data were obtained as averages of at least 3 measurements (Table 1).

The analyses performed showed average size heterogeneity in the sample, essentially due to the fact that the sonication process is a method that allowed only an approximate control of liposomal dimensions. The heterogeneity of the samples was confirmed by the value of the PDI that was much higher than 0.1 , indicating the presence of several dimensional populations of liposomes in each sample. The average dimension of liposomes did not vary significantly on hydrophilic molecule entrapping, whereas the embedding of Dex in the phospholipidic bilayer resulted in the formation of much smaller liposomes. This decrease was not unexpected because the insertion of a similar molecule, such as cholesterol, in the bilayer is known to affect the features of the membrane. A rigid steroid molecule may be able to stabilise the bilayer, making these structures more rigid and reducing their spontaneous leakage. In EPC liposomes, the insertion of cholesterol has been demonstrated to decrease the liposomal diameter (Takechi-Haraya et al., 2016). The value of $\zeta$-potential was slightly negative and did not vary very much in the investigated samples. However, attention is drawn to an increase of the negative value of $\zeta$-potential in the case of liposomes embedding Dex. This effect was expected, because Dex solubilises in the bilayer, inserting among the phospholipids like the structurally similar cholesterol. The presence of Dex, as demonstrated by Magarkar et al. for cholesterol (Magarkar et al., 2014), could decrease metal cation binding (i.e. $\mathrm{Na}^{+}$and $\mathrm{K}^{+}$) with the lipid headgroup, releasing these ions from the membrane interface to the aqueous bulk and decreasing the corresponding $\zeta$-potential value.

\section{DPSC immunophenotypic profile analysis}

To characterise the immunophenotypic profile of DPSCs exposed to liposomes, samples were analysed for the expression of a panel of surface markers characteristic of MSCs. As a matter of comparison, each CD marker level was referenced to the immunophenotype of routine sub-cultured cells at passage 5, namely T0 (Fig. 1). Briefly, about 95-99\% of DPSCs were stained positive for CD90, CD73, CD105 and CD29 and negative for CD45 (data not shown) with the highest MFI ratio assessed for CD90 and CD29 (373.10 and 96.83, respectively).
Likewise, CD73 and CD105 were clearly expressed, with MFI ratios of 62.94 and 16.08 (Fig. 1). The same surface marker panel was therefore applied to evaluate the immunophenotype of DPSCs cultured in DM and exposed to Lipo-Empty and Lipo-Mix for 3, 5 and $7 \mathrm{~d}$ (Fig. 2). As for CD90, it was broadly and time-dependently decreased with respect to $\mathrm{T} 0$ in all the experimental conditions. Lipo-Mix was the most effective formulation in lowering CD90 levels when compared to DM after only $3 \mathrm{~d}$ (278.20 and 322.84, respectively); however, being comparable to Lipo-Empty (276.21). Nevertheless, at $5 \mathrm{~d}$ of exposure to Lipo-Mix the CD90 level (MFI ratio 105.91) dramatically decreased compared to both Lipo-Empty (239.38) and DM (271.22). After $7 \mathrm{~d}$ of treatment, CD90 expression was homogeneously weak (Fig. 2). Conversely, CD73 was significantly expressed after $3 \mathrm{~d}$ in the presence of Lipo-Mix when compared to DM and Lipo-Empty (MFI ratios 230.06, 168.95 and 123.51, respectively) and this trend was maintained after 5 and $7 \mathrm{~d}$. As for CD105, its level was found to be higher than at T0 in all the experimental conditions, mainly after 3 and $5 \mathrm{~d}$. For instance, CD105 levels in DM and Lipo-Mix were comparable after $3 \mathrm{~d}$, whereas they were slightly but significantly increased after $5 \mathrm{~d}$ (Lipo-Mix 34.70 and DM 27.36). Finally, a peak in CD29 levels was registered in DPSC after $3 \mathrm{~d}$ of culture exposed to Lipo-Mix (258.18) with respect to DM (161.58) and Lipo-Empty (146.84). Likewise, CD29 was clearly expressed after $5 \mathrm{~d}$ when exposed to Lipo-Mix formulation and the expression was weakly, but significantly, maintained after $7 \mathrm{~d}$, compared to DM and Lipo-Empty (Fig. 2).

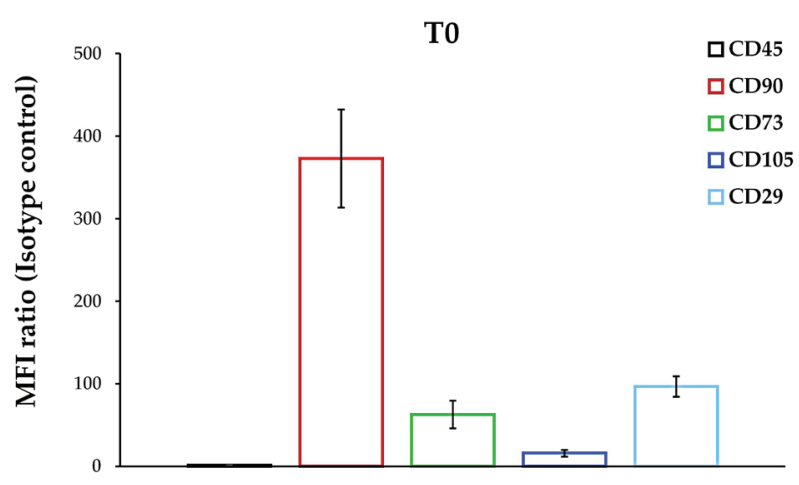

Fig. 1. Immunophenotypic profile of DPSCs under routine culture conditions (T0). Bars represent MFI ratios of CD45, CD90, CD73, CD105 and CD29 relative to the isotype control. Results from independent experiments $(n=3)$ are expressed as means \pm SD.

Table 1. Dimensions, PDI and $\zeta$-potential of the liposomal dispersions investigated.

\begin{tabular}{|c|c|c|c|}
\hline Samples & Average diameters $(\mathbf{n m})$ & PDI & $\zeta$-potential $(\mathbf{m V})$ \\
\hline Empty liposomes & $361 \pm 114$ & $0.26 \pm 0.03$ & $-6.65 \pm 0.44$ \\
\hline Liposomes + Asc $+\beta$-Gly & $398 \pm 139$ & $0.29 \pm 0.07$ & $-5.50 \pm 0.66$ \\
\hline Liposomes + Dex & $318 \pm 162$ & $0.34 \pm 0.02$ & $-10.33 \pm 0.32$ \\
\hline
\end{tabular}




\section{ECM deposition analysis}

In order to evaluate the ECM deposition, an ARS staining was carried out. The mineralised matrix deposition sites were distinctly red stained (Fig. 3). At early experimental times ( 1 and $3 \mathrm{~d}$ of culture), no matrix deposition could be identified in the LipoEmpty sample, a pale red staining was detectable in the presence of DM, whereas an extended mineralised matrix area could be clearly seen in the Lipo-Mix sample, thus indicating that the mineralisation of the ECM can be identified, starting from approximately $3 \mathrm{~d}$ of culture in Lipo-Mix and, to lesser extent, in DM samples. In Lipo-Empty and in DM samples large red stained areas, indicating the beginning of the ECM mineralisation, were detectable starting from $14 \mathrm{~d}$ of culture (Fig. 3).

\section{Osteogenic differentiation analysis}

To analyse the progression of the DPSC differentiation process, the ALP activity was measured in cell supernatants. The enzymatic activity appeared slightly but significantly augmented in Lipo-Mix sample with respect to both Lipo-Empty and DM samples after only $1 \mathrm{~d}$ of culture (1.24-fold increase). After $3 \mathrm{~d}$ of culture, the ALP activity recorded in Lipo-Mix still appeared to be clearly increased with respect to Lipo-Empty; at the same time ALP activity in DM sample was also statistically increased with

\section{$3 \mathrm{~d}$}
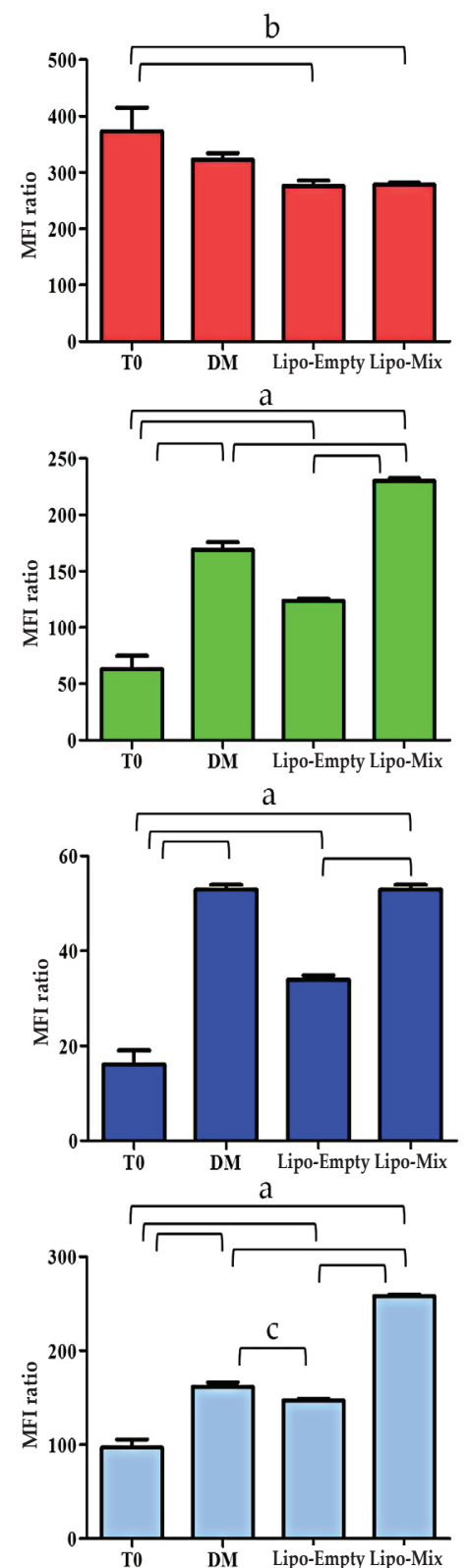

$5 \mathrm{~d}$
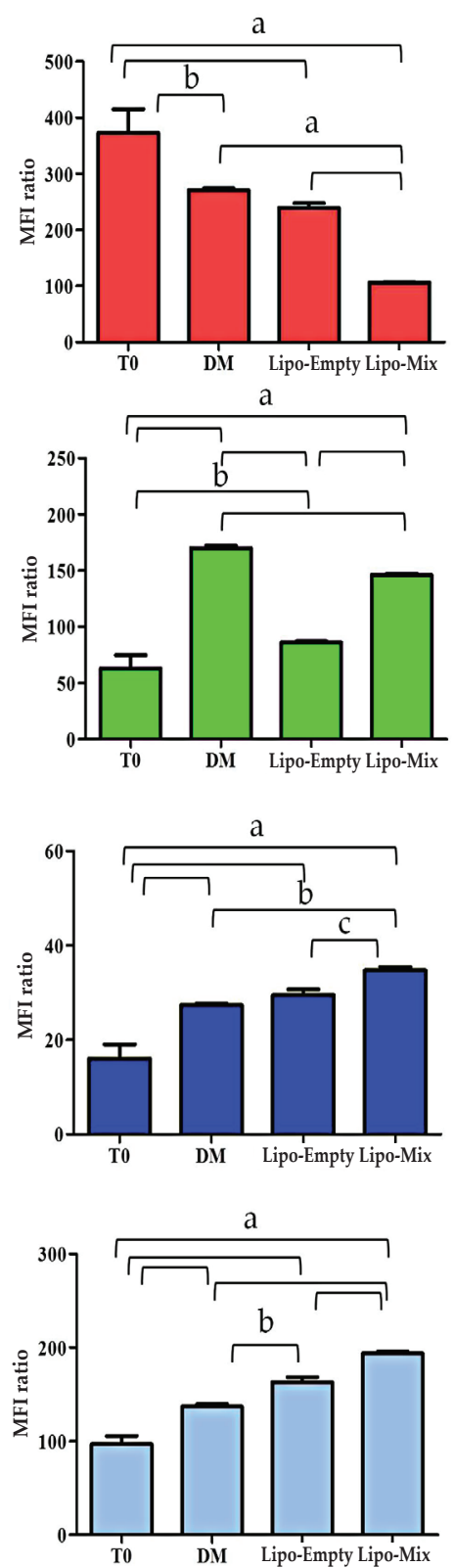

$7 \mathrm{~d}$
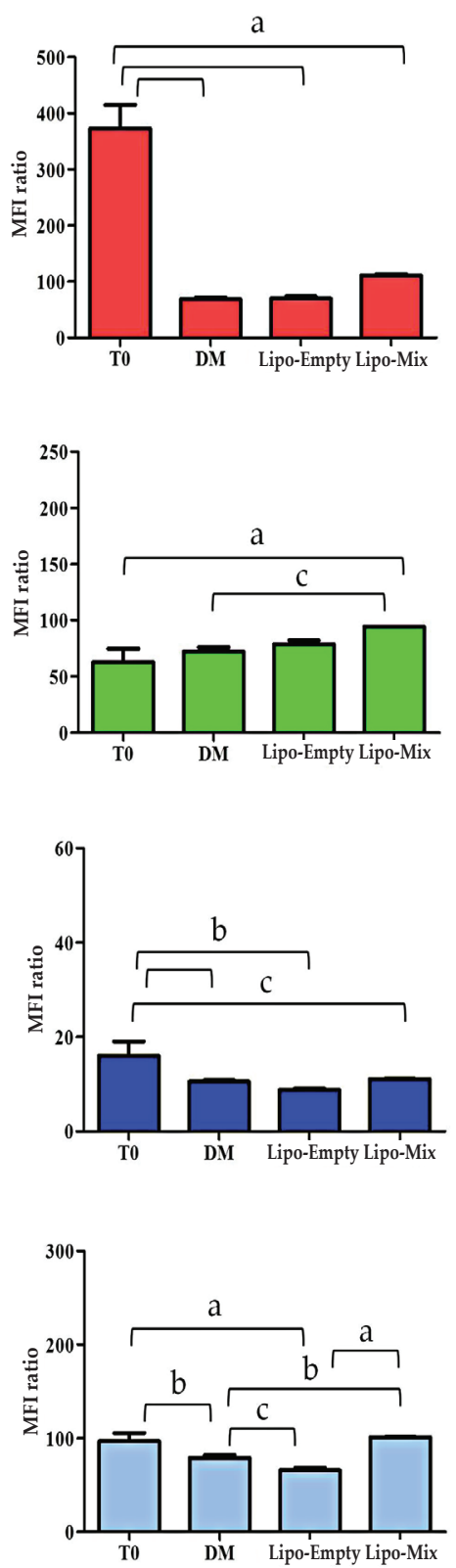

Fig. 2. Immunophenotypic profile of DPSCs exposed to DM, Lipo-Empty and Lipo-Mix after 3, 5 and $7 \mathrm{~d}$. Bars represent MFI ratios of CD45, CD90, CD73, CD105 and CD29 relative to the isotype control (FITC or PE). Results from independent experiments $(n=3)$ are expressed as means \pm SD. a: $p<0.0001, \mathrm{~b}: p<0.002, \mathrm{c}: p<0.05$. 
$1 \mathrm{~d}$
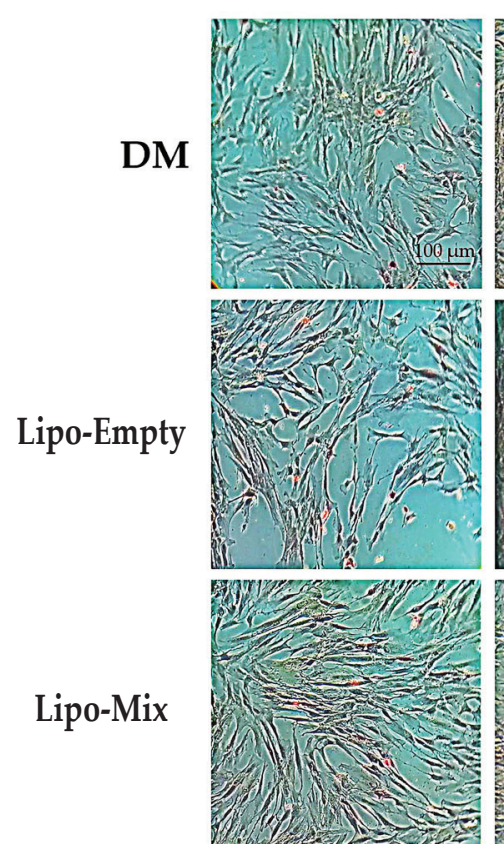

$3 \mathrm{~d}$
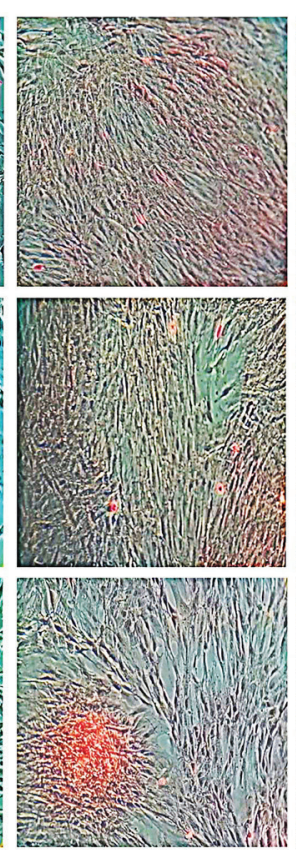

$7 \mathrm{~d}$
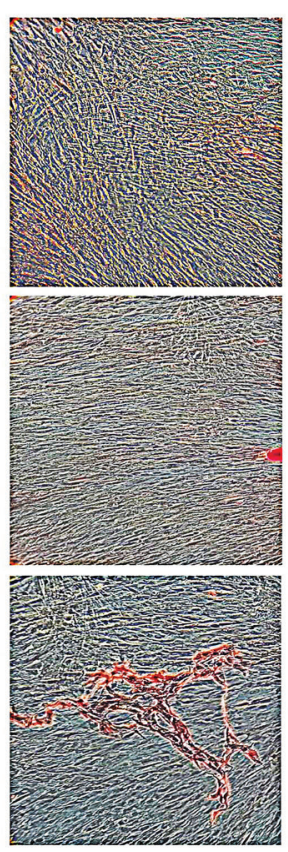

$14 \mathrm{~d}$
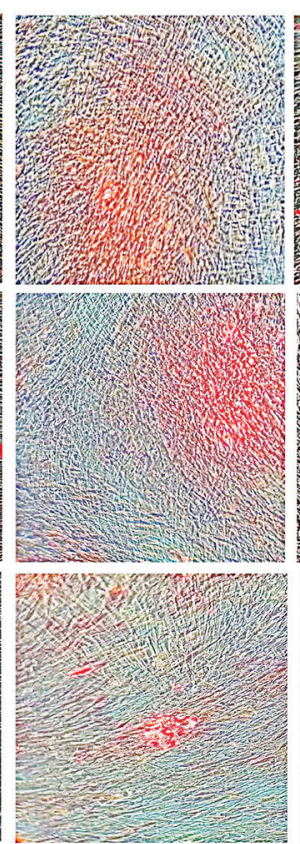

$21 \mathrm{~d}$
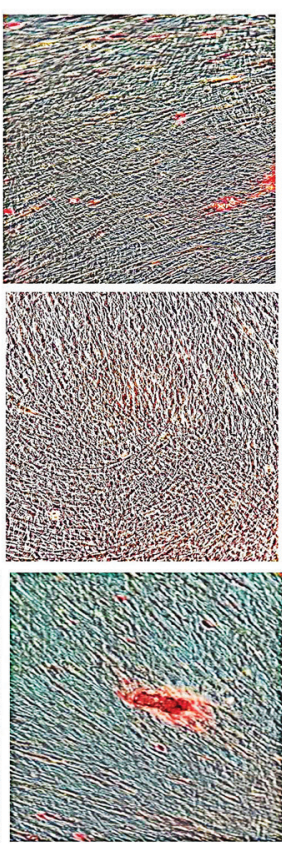

Fig. 3. ARS staining of mineralised bone matrix in DPSCs cultured up to $21 \mathrm{~d}$. Experimental times: 1, 3, 7, 14 and $21 \mathrm{~d}$; upper line: DPSCs cultured in DM. Central line: DPSCs cultured in presence of empty liposomes (Lipo-Empty ). Lower line: DPSCs cultured in presence of liposomes embedded with osteogenic differentiating factors (Lipo-Mix). Scale bar $=100 \mu \mathrm{m}$.

respect to Lipo-Empty; the same trend was recorded after $7 \mathrm{~d}$ of culture. After $21 \mathrm{~d}$ of culture, again, ALP activity in Lipo-Mix sample was significantly higher than that monitored for Lipo-Empty and DM samples (Fig. 4). To conclude, the expression of pro-COL1A1 and the secretion of Col1, a specific osteogenic differentiation marker, were evaluated by means of Western blot analysis and ELISA assay. Pro-COL1A1 appeared increased after $1 \mathrm{~d}$ of culture in the Lipo-Empty sample when compared to Lipo-Mix; after 3 d of culture this increase in LipoEmpty was converted in a high spike, which was shown to be statistically significant respect to both Lipo-Mix and DM experimental conditions. After $7 \mathrm{~d}$ of culture pro-COL1A1 expression in the LipoEmpty sample continued to be significantly higher with respect to Lipo-Mix. In parallel, an increase in protein expression was evidenced in the DM sample compared to Lipo-Empty and Lipo-Mix (Fig. 5). Col1 secretion in the culture medium, measured after $1,3,7,14$ and $21 \mathrm{~d}$ of culture, revealed that after $1 \mathrm{~d}$ of culture a statistically significant higher level secretion was detected in the DM sample $(6.22 \mu \mathrm{g})$ with respect to both Lipo-Empty $(2.27 \mu \mathrm{g})$ and Lipo-Mix $(3.10 \mu \mathrm{g})$. After $3 \mathrm{~d}$, the absolute highest value of the experimental set was registered in the Lipo-Mix sample $(17.15 \mu \mathrm{g})$. Nevertheless, in the presence of Lipo-Empty Col1 secretion was distinctly increased $(6.43 \mu \mathrm{g})$, whereas DPSCs cultured only in DM secreted $4.65 \mu \mathrm{g}$ of Col1. Starting from day 3 up to 21 days, the highest statistically significant Col1 secretion level was recorded in the Lipo-Mix sample with respect to the Lipo-Empty and DM at $7 \mathrm{~d}$ of culture $(4.59 \mu \mathrm{g}, 1.12 \mu \mathrm{g}$ and $1.74 \mu \mathrm{g}$, respectively) (Fig. 6).

The osteoblastic marker BSP II was also evaluated by means of Western blot analysis. A statistically significant increase in protein expression was seen in the DM sample with respect to Lipo-Empty and Lipo-Mix after $1 \mathrm{~d}$ of culture. The same trend was detectable after 3 and $7 \mathrm{~d}$ of culture (Fig. 7).

\section{Oxidative stress evaluation}

The oxidative stress occurrence was also taken into consideration by quantifying the $\mathrm{H}_{2} \mathrm{O}_{2}$ concentration

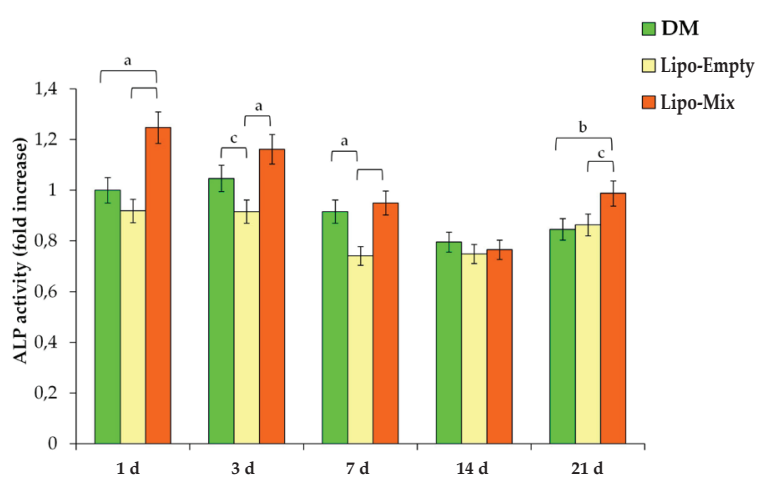

Fig. 4. Quantitative determination of the ALP activity levels in DPSCs cultured in DM, in presence of Lipo-Empty and Lipo-Mix for 1, 3, 7, 14 and $21 \mathrm{~d}$. Values represent the means $\pm \mathrm{SD}(n=3)$. The bar graph shows the enzymatic activity of ALP in cell supernatants as the fold increase on the DM sample at day 1. a: $p<0.0001, \mathrm{~b}: p<0.005$, c: $p<0.01$. 
within the culture medium (Fig. 8a). In the Lipo-Mix samples, the hydrogen peroxide pool decreased over $7 \mathrm{~d}$ of culture, where the concentration was extremely low with respect to that in DM and Lipo-Empty (555.65, 1490.96 and $1000.92 \mathrm{ng} / \mathrm{mL}$, respectively). Notably, after $21 \mathrm{~d}$ of culture in the presence of
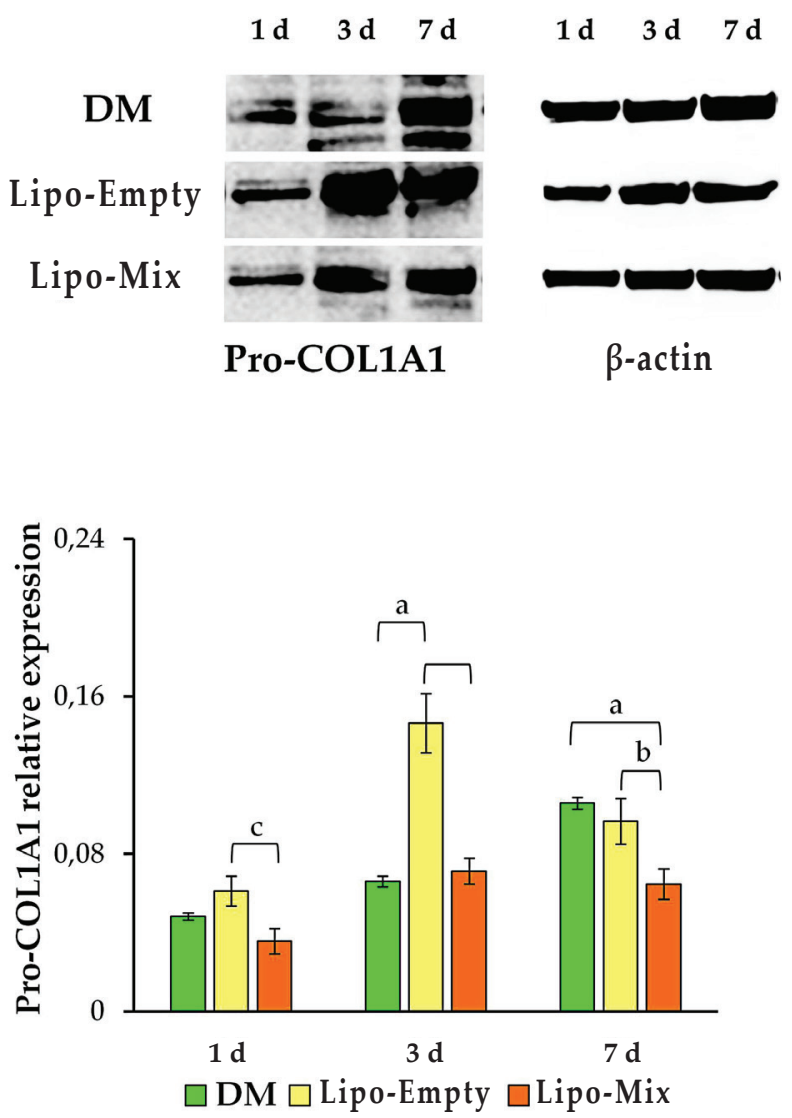

Fig. 5 Western blot analysis of pro-COL1A1 expression in DPSCs cultured in DM, in presence of Lipo-Empty and Lipo-Mix for 1, 3 and $7 \mathrm{~d}$. The most representative of 3 separate experiments is shown. Densitometric values $( \pm S D)$ are expressed as the mean IOI, normalised on $\beta$-actin. a: $p<0.0001$, b: $p<0.005$, c: $p<0.01$.

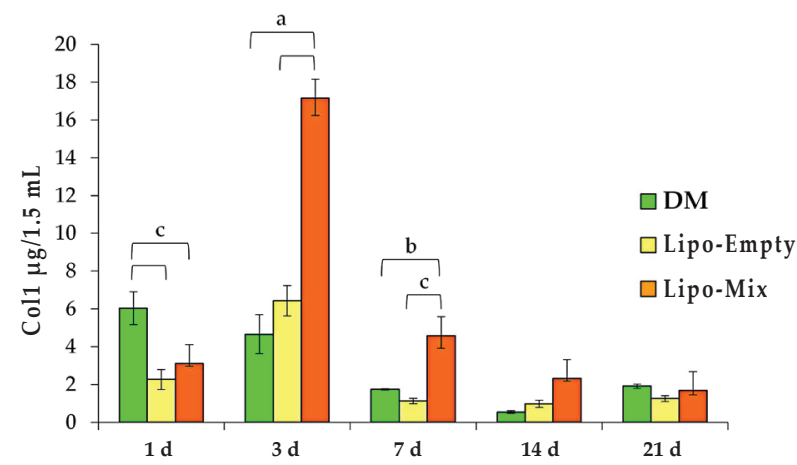

Fig. 6. ELISA assay for Col1 secretion in DPSCs cultured in DM, in presence of Lipo-Empty and Lipo-Mix for 1, 3, 7, 14 and $21 \mathrm{~d}$. Secretion levels are reported as $\mu \mathrm{g} / 1.5 \mathrm{~mL}$ (total volume of supernatant in the well). Values represent the means $\pm \mathrm{SD}(n=3)$. a: $p<0.0001$, b: $p<0.005$, c: $p<0.01$.
Lipo-Mix, the lowest absolute value was registered $(285.88 \mathrm{ng} / \mathrm{mL})$. In parallel, the activation of the protein Erk1/2 was estimated by means of Western blot analysis after 1, 3 and $7 \mathrm{~d}$ of culture. After $1 \mathrm{~d}$ of culture a statistically significant almost 2 - and 3 -foldincrease of the $p$ Erk $1 / 2$ : Erk $1 / 2$ ratio was detected in Lipo-Mix with respect to both DM and Lipo-Empty samples, respectively; DM pErk1/2 : Erk1/2 ratio was also significantly augmented compared to the LipoEmpty ratio. After $3 \mathrm{~d}$ of culture a trend inversion, compared to day 1 , could be identified. pErk1/2 : Erk1/2 ratio in fact appeared statistically increased in the DM sample with respect to Lipo-Mix and Lipo-Empty experimental conditions. Moreover, Erk activation in the Lipo-Mix sample was significantly augmented with respect to the Lipo-Empty. The same trend for $\mathrm{pErk} 1 / 2$ : Erk1/2 ratio was found after $7 \mathrm{~d}$ of culture (Fig. 8b).

\section{Discussion}

MSCs derived from dental pulp are critical components required for the regeneration of soft and hard tissues, thus playing a pivotal role in tissue engineering and regenerative medicine. Considering that DPSC differentiation towards the osteoblastic phenotype could be enhanced and speeded up by
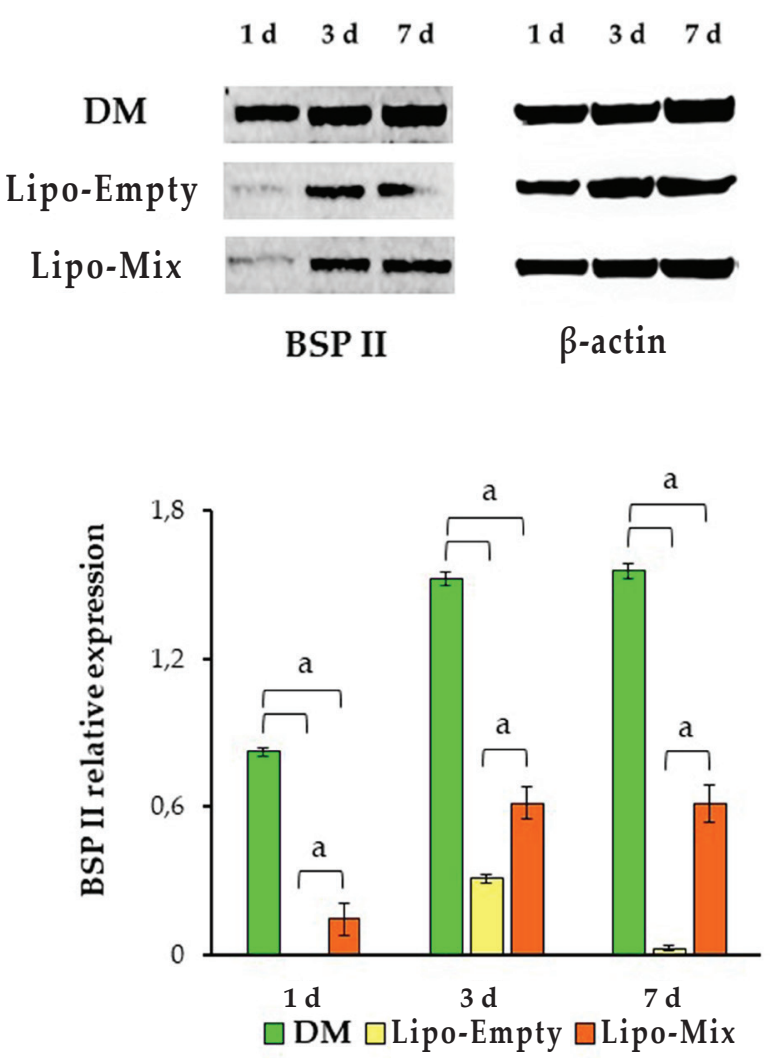

Fig. 7. Western blot analysis of BSPII expression in DPSCs cultured in DM, in presence of LipoEmpty and Lipo-Mix for 1, 3 and $7 \mathrm{~d}$. The most representative of three separate experiments is shown. Densitometric values $( \pm \mathrm{SD})$ are expressed as the mean IOI, normalised on $\beta$-actin. a: $p<0.0001$. 
delivering in situ specific differentiating factors (Melling et al., 2018), differentiating factors were embedded within liposomes and DPSCs then exposed to these constructs. DPSCs were chosen as the experimental model, based on the evidence that they represent an appreciable cell source for hardtissue regeneration (Otabe et al., 2012). Dental pulp contains a mesenchymal population expressing key MSC-associated markers. Remarkable expressionlevels of CD90, CD73, CD105 and CD29 represent the minimal criteria for defining a multipotent mesenchymal stromal cell (Dominici et al., 2006). CD90 is a GPI-anchored cell surface protein that plays multiple roles in many physiological processes, such as cell differentiation and regeneration (Yang et al., 2020). It has been reported that a CD90 knockdown enhances the osteogenic differentiation of MSCs in vitro (Moraes et al., 2016) and plays a crucial role in MSC fate decision (Saalbach and Anderegg, 2019). Notably, in the current experimental model, the lowest level of CD90 expression was registered after $7 \mathrm{~d}$ with Lipo-Mix, making it plausible to hypothesise an osteogenic commitment enhancement with respect to the canonical DM. The ecto-5'-nucleotidase, also known as CD73, is a GPI-anchored cell surface protein involved in adenosine production. It has been reported that the extracellular nucleotide ATP can be one of the key mediators in bone metabolism (Takedachi et al., 2012) and that adenosine-A1 receptor stimulation enhances osteogenic differentiation of DPSCs through the Wnt/ $\beta$-catenin signalling (D'Alimonte et al., 2013). Thus, highly significant CD73 levels of expression in DPSCs after only $3 \mathrm{~d}$ exposed to Lipo-Mix, could be related to an early induction of the osteogenic differentiation (Fig. 2). CD29, is a transmembrane protein that can interact with matrix proteins and collagen fibres generating intracellular signals and can be targeted to promote homing of MSCs to bone, osteogenic differentiation and bone formation (Marie, 2013). In the presence of Lipo-Mix, CD29 was significantly expressed by DPSCs (Fig. 2), suggesting enhanced ECM trafficking. To conclude, levels of CD105, a transmembrane glycoprotein functioning as a co-receptor for ligands of the TGF- $\beta$ superfamily (Schoonderwoerd et al., 2020), has been found to increase rapidly in freshly harvested MSCs after 4-7 d of culture and it afterwards gradually decreases during differentiation (Levi et al.,

a

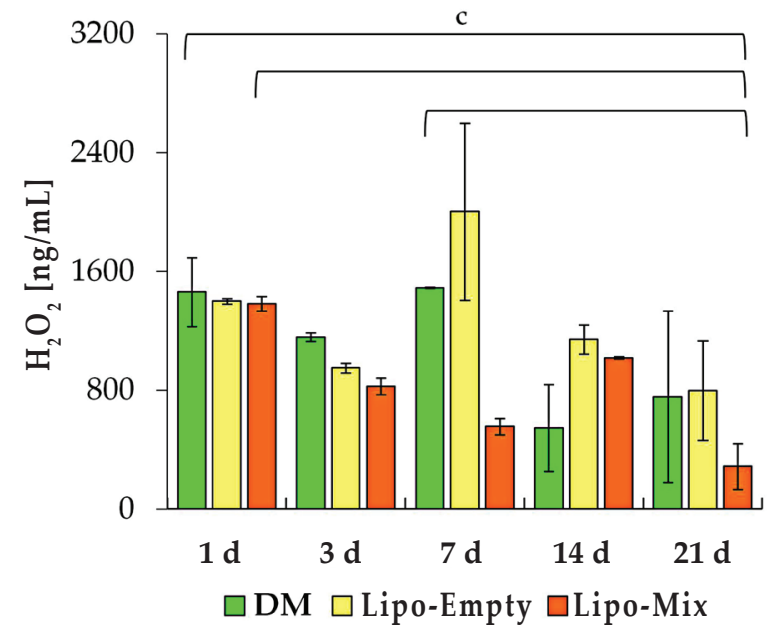

b
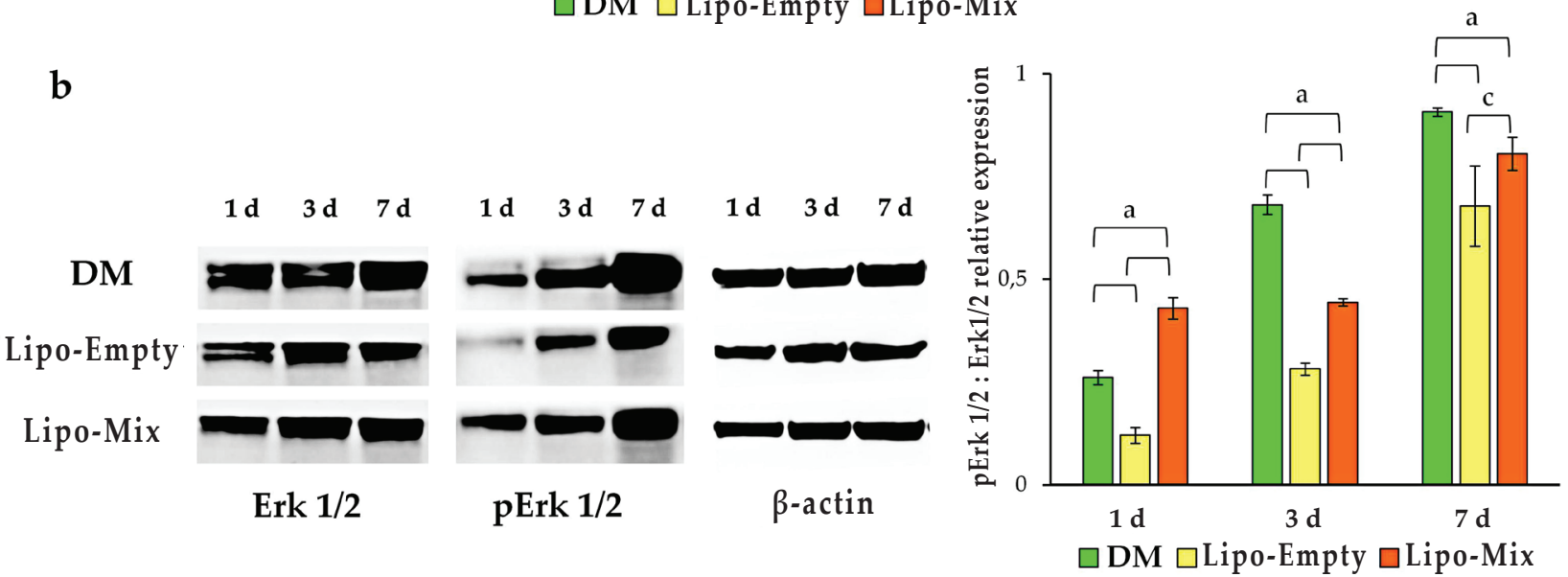

Fig. 8. Oxidative stress occurrence and related proteins in DPSCs cultured in DM, in presence of Lipo-Empty and Lipo-Mix. (a) Quantitative determination of the $\mathrm{H}_{2} \mathrm{O}_{2}$ released. Values represent the means $\pm \mathrm{SD}(n=3)$. The bar graph shows the $\mathrm{H}_{2} \mathrm{O}_{2}$ release $(\mathrm{ng} / \mathrm{mL})$ in cell supernatants. c: $p<0.005$. (b) Western blot analysis of ERK1/2 and p-ERK1/2 expression in DPSCs cultured in DM, in presence of Lipo-Empty and Lipo-Mix for 1, 3 and $7 \mathrm{~d}$. The most representative of three separate experiments is shown. Densitometric values $( \pm$ SD) are expressed as the mean of IOI, normalised on $\beta$-actin. a: $p<0.0002$, c: $p<0.005$. 
2011). The results of the current study, regarding the CD105 trend in DPSC s exposed to both DM and Lipo-Mix, were perfectly in agreement with this evidence (Fig. 2). Moreover, the hypothesis that the CD105 peaks after 3 and $5 \mathrm{~d}$ of culture coincide with an early induction of the osteogenic differentiation in the Lipo-Mix condition was confirmed by the matrix deposition. Clusters of calcium and phosphate salts can be detected early in the Lipo-Mix, whereas an appreciable mineralisation can only be seen in the DM and Lipo-Empty conditions at late stages (Fig. 3).

The osteogenic differentiation progress in the 3 tested experimental conditions was also measurable from the ALP activity. Firstly, these data again provided evidence for a stronger and faster progression of osteogenic commitment in the LipoMix condition. Secondly, given that MSCs are able to differentiate into both osteoblasts and adipocytes, and that the balance between adipogenesis and osteogenesis moves in favour of adipogenesis when cells are exposed to liposome formulations (James, 2013), definitively excludes the possibility of empty liposomes triggering an osteogenic differentiation (Fig. 4).

ROS are oxygen-derived small molecules, which react readily with a variety of chemical structures such as proteins, lipids, sugars, and nucleic acids. It is currently believed that regulated ROS production promotes essential signalling pathways, which regulate cell functions such as differentiation, and numerous reports describe the importance of a redox control of MSC differentiation (Kim et al., 2020; Sancilio et al., 2019;). In addition, ROS-activated MAPK signalling can regulate stem cell proliferation, migration and differentiation (Kong et al., 2019). It is plausible to assume that a redox control through pErk1/2 occurs under the osteogenic commitment of DPSCs after a Lipo-Mix administration, being the $\mathrm{H}_{2} \mathrm{O}_{2}$ concentration decreased in a time-dependent manner and Erk1/2 activated by phosphorylation in parallel. This result is in agreement with Mody et al., who demonstrated the ability of $\mathrm{H}_{2} \mathrm{O}_{2}$ to inhibit bone differentiation (Mody et al., 2001). Prominently, LipoMix seemed to trigger and shorten this molecular pathway with respect to DM, once again supporting the hypothesis that POPC liposomes can be efficient tools for differentiating-factor delivery.

Col1 is a key protein of the bone ECM able to bind cell-surface integrins, such as CD29, and to promote osteoblast adhesion and differentiation. Furthermore, Col1 can be considered an early marker of osteogenic differentiation, being an upstream activator of Runx2 transcription factor recruitment (Di Carlo et al., 2020; Lagenbach and Handschel, 2013; Pavlin et al., 2001). The experimental model for the current study reflects this work. In fact, the Lipo-Empty condition showed a tentative hyper-expression of pro-Col1 that did not correspond to a higher secretion of Col1 in the culture medium. This suggested that the Lipo-Empty was unable to actually promote DPSC osteoblastic differentiation. In contrast, under the Lipo-Mix condition, pro-Col1 is synthesised, converted to the mature form of Col1 and secreted into the culture medium, allowing a very high level of secretion to be recorded at the early stages of the culture - confirming Col1's role as an early marker of the osteogenic differentiation (Fig. 6).

Furthermore, the Baht et al. (2008) and Tye et al. (2005) groups demonstrated that the efficiency of BSP II in HA nucleation is significantly strengthened in presence of Col1 - implying a co-operative effect of the 2 proteins in mineral deposition. In the light of this evidence, the lower expression of BSP II in LipoMix-treated cells can be argued considering that the very high availability of Col1, needed for the early HA nodule formation, keeps the BSP II protein expression at basal level: however, guaranteeing a high level of mineral deposition (Fig. 7). There is a plethora of literature reporting that MSCs play an essential role in supporting bone healing through the secretion of nutritional and immunomodulatory factors and that the MSC-based therapy can influence bone regeneration through both paracrine and autocrine systems (Xu et al., 2020). In view of this, trying to enhance DPSC commitment to the osteogenic lineage with the Lipo-Mix formulation could be a suitable tool for regenerative medicine purposes in a clinical situation. Likewise, investigating the intracellular molecular pathway triggered after its administration lays the ground for further tissue engineering and drug delivery studies in an effort to design appropriate coatings or supplementations carried by liposomes.

\section{Conclusions}

The results presented demonstrate that Lipo-Mix is capable of shortening DPSC osteogenic commitment in terms of CD-marker expression and ALP activity, when compared to the canonical DM. At a molecular level, the matrix mineralisation enhancement can be partly ascribed to BSPII-collagen interaction, due to the large amount of Col1 secreted in the presence of Lipo-Mix. This new formulation positively modulates ROS and proteins related to survival. Further analyses are required to clarify the molecular mechanisms underlying Lipo-Mix administration and its better performance in terms of mineralisation and differentiation in vitro.

The Lipo-Mix formulation could represent an innovative and feasible form to deliver both hydrophilic and lipid molecules directly to DPSCs. They could be encapsulated within the same structure, represented by the outer phospholipidic bilayer and the liposomal aqueous core, depending on the solubility features of the differentiating factors. Taken together, the results of the current study provide the foundation for constructing innovative and economical tools to enhance and to shorten MSC commitment to the osteoblastic lineage for regenerative medicine purposes. 


\section{Acknowledgements}

This work was supported by the Italian Ministry of University grants (Fondi FAR) assigned to Prof. Amelia Cataldi (2019 and 2020), to Dr. Susi Zara (2019 and 2020) and to Dr. Marialucia Gallorini (2020), and by Programma Operativo Nazionale (PON) Ricerca e Innovazione 2014-2020 - Fondo Sociale Europeo, Azione I.2 "Attrazione e Mobilità Internazionale dei Ricercatori".

\section{References}

Akbarzadeh A, Rezaei-Sadabady R, Davaran S, Joo SW, Zarghami N, Hanifehpour Y, Samiei M, Kouhi M, Nejati-Koshki K (2013) Liposome: classification, preparation, and applications. Nanoscale Res Lett 8: 102. DOI: 10.1186/1556-276X-8-102.

Almeida AJ, Souto E (2007) Solid lipid nanoparticles as a drug delivery system for peptides and proteins. Adv Drug Deliv Rev 59: 478-490.

Atashi F, Modarressi A, Pepper MS (2015) The role of reactive oxygen species in mesenchymal stem cell adipogenic and osteogenic differentiation: a review. Stem Cells Dev 24: 1150-1163.

Baht GS, Hunter GK, Goldberg HA (2008) Bone sialoprotein-collagen interaction promotes hydroxyapatite nucleation. Matrix Biol 27: 600-608.

Bangham AD, Standish MM, Watkins JC (1965) Diffusion of univalent ions across the lamellae of swollen phospholipids. J Mol Biol 13: 238-252.

Brożek R. Kurpisz M, Koczorowski R (2017) The oral cavity - potential source of stem cells. Postepy Hig Med Dosw (Online). 71: 881-894.

Cortese K, Marconi S, Aiello C, Gagliani MC, Pilato S, Zappacosta R, Fontana A, Castagnola P (2020) Liposomes loaded with the proteasome inhibitor z-leucinyl-leucinyl-norleucinal are effective in inducing apoptosis in colorectal cancer cell lines. Membranes (Basel) 91: 1-11.

D’Alimonte I, Lannutti A, Pipino C, Di Tomo P, Pierdomenico L, Cianci E, Antonucci I, Marchisio M, Romano M, Stuppia L, Caciagli F, Pandolfi A, Ciccarelli R (2013) Wnt signaling behaves as a "master regulator" in the osteogenic and adipogenic commitment of human amniotic fluid mesenchymal stem cells. Stem Cell Rev Rep 9: 642-654.

Di Carlo R, Di Crescenzo A, Pilato S, Ventrella A, Piattelli A, Recinella L, Chiavaroli A, Giordani S, Baldrighi M, Camisasca A, Zavan B, Falconi M, Cataldi A, Fontana A, Zara S (2020) Osteoblastic differentiation on graphene oxide-functionalized titanium surfaces: an in vitro study. Nanomaterials (Basel) 10: 654. DOI: 10.3390/nano10040654.

Dominici M, Le Blanc K, Mueller I, SlaperCortenbach I, Marini F, Krause D, Deans R, Keating A, Prockop Dj, Horwitz E (2006) Minimal criteria for defining multipotent mesenchymal stromal cells. The International Society for Cellular Therapy position statement. Cytotherapy 8: 315-317.
Fahy E, Subramaniam S, Murphy RC, Nishijima M, Raetz CR, Shimizu T, Spener F, van Meer G, Wakelam MJ, Dennis EA (2009) Update of the LIPID MAPS comprehensive classification system for lipids. J Lipid Res 50 (Suppl): S9-S14.

Gomes-da-Silva LC, Santos AO, Bimbo LM, Moura V, Ramalho JS, Pedroso de Lima MC, Simões S, Moreira JN (2012) Toward a siRNA-containing nanoparticle targeted to breast cancer cells and the tumor microenvironment. Int J Pharm 434: 9-19.

Goto N, Fujimoto K, Fujii S, Ida-Yonemochi H, Ohshima H, Kawamoto T, Noshiro M, Shukunami C, Kozai K, Kato Y (2016) Role of MSX1 in osteogenic differentiation of human dental pulp stem cells. Stem Cells Int 2016: 8035759. DOI: 10.1155/2016/8035759.

Graziano A, d'Aquino R, Laino G, Papaccio G (2008) Dental pulp stem cells: a promising tool for bone regeneration. Stem Cell Rev 4: 21-26.

James AW (2013) Review of signaling pathways governing MSC osteogenic and adipogenic differentiation. Scientifica (Cairo) 2013: 684736. DOI: 10.1155/2013/684736.

Kim DH, Kim DH, Heck BE, Shaffer M, Hur J, Yoo $\mathrm{KH}$ (2020) A natural supplement formula reduces antioxidative stress and enhances osteo-chondrogenic differentiation potential in mesenchymal stem cells. J Clin Biochem Nutr 66: 206-212.

Kong Y, Hu X, Zhong Y, Xu K, Wu B, Zheng J (2019) Magnesium-enriched microenvironment promotes odontogenic differentiation in human dental pulp stem cells by activating ERK/BMP2/ Smads signaling. Stem Cell Res Ther 10: 378-389.

Langenbach F, Handschel J (2013) Effects of dexamethasone, ascorbic acid and $\beta$-glycerophosphate on the osteogenic differentiation of stem cells in vitro. Stem Cell Res Ther 4: 117-124.

Lee K, Silva EA, Mooney DJ (2011) Growth factor delivery-based tissue engineering: general approaches and a review of recent developments. J R Soc Interface 8: 153-170.

Levi B, Wan DC, Glotzbach JP, Hyun J, Januszyk M, Montoro D, Sorkin M, James AW, Nelson ER, Li S, Quarto N, Lee M, Gurtner GC, Longaker MT (2011) CD105 protein depletion enhances human adiposederived stromal cell osteogenesis through reduction of transforming growth factor $\beta 1$ (TGF- $\beta 1$ ) signaling. J Biol Chem 286: 39497-39509.

Magarkar A, Dhawan V, Kallinteri P, Viitala T, Elmowafy M, Róg T, Bunker A (2014) Cholesterol level affects surface charge of lipid membranes in saline solution. Sci Rep 4: 5005-5010.

Maleki M, Ghanbarvand F, Reza Behvarz M, Ejtemaei M, Ghadirkhomi E (2014) Comparison of mesenchymal stem cell markers in multiple human adult stem cells. Int J Stem Cells 7: 118-126.

Marie PJ (2013) Targeting integrins to promote bone formation and repair. Nat Rev Endocrinol 9: 288-295.

Melling GE, Colombo JS, Avery SJ, Ayre WN, Evans SL, Waddington RJ, Sloan AJ (2018) Liposomal 
delivery of demineralized dentin matrix for dental tissue regeneration. Tissue Eng Part A 24: 1057-1065.

Mody N, Sarafian TA, Demer L (2001) Oxidative stress modulates osteoblastic differentiation of vascular and bone cells. Free Radic Biol Med 3: 509519.

Monteiro N, Martins A, Ribeiro D, Faria S, Fonseca NA, Moreira JN, Reis RL, Neves NM (2015) On the use of dexamethasone-loaded liposomes to induce the osteogenic differentiation of human mesenchymal stem cells. J Tissue Eng Regen Med 9: 1056-1066.

Moraes DA, Sibov TT, Pavon LF, Alvim PQ, Bonadio RS, Da Silva JR, Pic-Taylor A, Toledo OA, Marti LC, Azevedo RB, Oliveira DM (2016) A reduction in CD90 (THY-1) expression results in increased differentiation of mesenchymal stromal cells. Stem Cell Res Ther 7: 97-111.

Moreira JN, Ishida T, Gaspar R, Allen TM (2002) Use of the post-insertion technique to insert peptide ligands into pre-formed stealth liposomes with retention of binding activity and cytotoxicity. Pharm Res 19: 265-269.

Moura V, Lacerda M, Figueiredo P, Corvo ML, Cruz ME, Soares R, de Lima MC, Simões S, Moreira JN (2012) Targeted and intracellular triggered delivery of therapeutics to cancer cells and the tumor microenvironment: impact on the treatment of breast cancer. Breast Cancer Res Treat 133: 61-73.

Otabe K, Muneta T, Kawashima N, Suda H, Tsuji K, Sekiya I (2012) Comparison of gingiva, dental pulp, and periodontal ligament cells from the standpoint of mesenchymal stem cell properties. Cell Med 4: 13-21.

Pavlin D, Zadro R, Gluhak-Heinrich J (2001) Temporal pattern of stimulation of osteoblastassociated genes during mechanically-induced osteogenesis in vivo: early responses of osteocalcin and type I collagen. Connect Tissue Res 42: 135-148.

Saalbach A, Anderegg U (2019) Thy-1: more than a marker for mesenchymal stromal cells. FASEB J 33: 6689-6696.

Sancilio S, Marsich E, Schweikl H, Cataldi A, Gallorini M (2019) Redox control of IL-6-mediated dental pulp stem-cell differentiation on alginate/ hydroxyapatite biocomposites for bone ingrowth. Nanomaterials (Basel) 9: 1656-1668.

Santo VE, Gomes ME, Mano JF, Reis RL (2013a) Controlled release strategies for bone, cartilage, and osteochondral engineering - Part I: recapitulation of native tissue healing and variables for the design of delivery systems. Tissue Eng Part B Rev 19: 308-326.

Santo VE, Gomes ME, Mano JF, Reis RL (2013b) Controlled release strategies for bone, cartilage, and osteochondral engineering - Part II: challenges on the evolution from single to multiple bioactive factor delivery. Tissue Eng Part B Rev 19: 327-352.

Schoonderwoerd MJA, Goumans MTH, Hawinkels LJAC (2020) Endoglin: beyond the endothelium. Biomolecules 10: 289-306.

Singh AK, Cummings EB, Throckmorton DJ (2001) Fluorescent liposome flow markers for microscale particle-image velocimetry. Anal Chem 73: 1057-1061.
Sinjari B, Pizzicannella J, D' Aurora M, Zappacosta R, Gatta V, Fontana A, Trubiani O, Diomede F (2019) Curcumin/liposome nanotechnology as delivery platform for antinflammatory activities via NFkB/ ERK/pERK pathway in human dental pulp treated with 2-hydroxyethyl methacrylate (HEMA). Front. Physiol 10: 633-644.

Song IH, Caplan AI, Dennis JE (2009) In vitro dexamethasone pretreatment enhances bone formation of human mesenchymal stem cells in vivo. J Orthop Res 27: 916-921.

Takechi-Haraya Y, Sakai-Kato K, Abe Y, Kawanishi T, Okuda H, Goda Y (2016) Atomic force microscopic analysis of the effect of lipid composition on liposome membrane rigidity. Langmuir 32: 6074-6082.

Takedachi M, Oohara H, Smith BJ, Iyama M, Kobashi M, Maeda K, Long CL, Humphrey MB, Stoecker BJ, Toyosawa S, Thompson LF, Murakami S (2012) CD73-generated adenosine promotes osteoblast differentiation. J Cell Physiol 227: 26222631.

Tye CE, Hunter GK, Goldberg HA (2005) Identification of the type I collagen-binding domain of bone sialoprotein and characterization of the mechanism of interaction. J Biol Chem 280: 1348713492.

Viale M, Fontana A, Maric I, Monticone M, Angelini G, Gasbarri C (2016) Preparation and antiproliferative activity of liposomes containing a combination of cisplatin and procainamide hydrochloride. Chem Res Toxicol 29: 1393-1395.

Xu GP, Zhang XF, Sun L, Chen EM (2020) Current and future uses of skeletal stem cells for bone regeneration. World J Stem Cells 12: 339-350.

Yang J, Zhan XZ, Malola J, Li ZY, Pawar JS, Zhang HT, Zha ZG (2020) The multiple roles of Thy-1 in cell differentiation and regeneration. Differentiation 113: 38-48.

Zappacosta R, Aschi M, Ammazzalorso A, Di Profio P, Fontana A, Siani G (2019a) Embedding calix[4]resorcinarenes in liposomes: experimental and computational investigation of the effect of resorcinarene inclusion on liposome properties and stability. Biochim Biophys Acta Biomembr 1861: 12521259.

Zappacosta R, Cornelio B, Pilato S, Siani G, Estour F, Aschi M, Fontana A (2019b). Effect of the incorporation of functionalized cyclodextrins in the liposomal bilayer. Molecules 24: 1387. DOI: 10.3390/ molecules24071387.

Zappacosta R, Siani G, Silvi S, Credi A, Fontana A (2014) Light-driven directed proton transport across the liposomal membrane. Langmuir 30: 13667-13672.

\section{Discussion with Reviewer}

Reviewer 1: In my opinion, to reinforce the conclusions, the authors should perform in vivo assay using liposomes to induce bone regeneration in murine model with mandible damage. 
Authors: The referee is correct in stating that the liposomal formulations should be tested in a murine in vivo model with mandible damage. However, as the liposomal formulation and the experimental model used for delivering the osteogenic differentiating factors to DPSCs is extremely new, the current study was very necessary in order to investigate the basal molecular mechanisms underlying these biological processes. This paper only represents the starting point of a bigger project, in which in vivo studies are planned to be included.

Editor's note: The Scientific Editor responsible for this paper was Thimios Mitsiadis. 